\title{
El registro de Pelagornithidae (Aves: Pelecaniformes) y la avifauna neógena del Pacífico sudeste
}

Le registre de Pelagornithidae (Oiseaux: Pelecaniformes) et l'avifaune néogène du Sud-est Pacifique

The record of Pelagorninthidae (Aves: Pelecaniformes) and the Neogene avifauna of the southeast Pacific

Martín Chávez, Marcelo Stucchi y Mario Urbina

\section{OpenEdition \\ Journals}

Edición electrónica

URL: http://journals.openedition.org/bifea/3780

DOI: $10.4000 /$ bifea. 3780

ISSN: $2076-5827$

Editor

Institut Français d'Études Andines

Edición impresa

Fecha de publicación: 1 agosto 2007

Paginación: 175-197

ISSN: 0303-7495

Referencia electrónica

Martín Chávez, Marcelo Stucchi y Mario Urbina, «El registro de Pelagornithidae (Aves:

Pelecaniformes) y la avifauna neógena del Pacífico sudeste », Bulletin de l'Institut français d'études

andines [En línea], 36 (2) | 2007, Publicado el 01 febrero 2008, consultado el 01 diciembre 2020. URL

http://journals.openedition.org/bifea/3780 ; DOI : https://doi.org/10.4000/bifea.3780

\section{(c) (i) $\odot$}

Les contenus du Bulletin de l'Institut français d'études andines sont mis à disposition selon les termes de la licence Creative Commons Attribution - Pas d'Utilisation Commerciale - Pas de Modification 4.0 International. 


\title{
El registro de Pelagornithidae (Aves:Pelecaniformes) y la avifauna neógena del Pacífico sudeste
}

\author{
Martín Chávez* \\ Marcelo Stucchi** \\ Mario Urbina***
}

\section{Resumen}

Se examina el registro neógeno de la extinta familia Pelagornithidae en las Formaciones Pisco (Perú) y Bahía Inglesa (Chile) en la costa pacífica de América del Sur. Se reportan nuevos especímenes pertenecientes al género Pelagornis, procedentes de los niveles Sacaco y Aguada de Lomas de la Formación Pisco, y del nivel fosfático de la Formación Bahía Inglesa. Asimismo se presentan elementos craneales y postcraneales de género indeterminado del nivel Montemar de la Formación Pisco y de la base de la misma, límite entre el nivel Cerro la Bruja y la Formación Chilcatay. Se compara el presente registro con los últimos antecedentes conocidos para la familia en el hemisferio sur. Se discute la problemática taxonómica del grupo y la correcta asignación de elementos fósiles a la familia. Se comenta sobre el actual conocimiento de la avifauna neógena dentro del área de estudio.

Palabras clave: Pelagornithidae, Formación Pisco, Formación Bahía Inglesa, neógeno

* Instituto de Zoología, Universidad Austral (Valdivia, Chile). E-mail: paleoaeolos@gmail.com

** Asociación para la Investigación y Conservación de la Biodiversidad (AICB, Perú). Becario del Instituto Francés de Estudios Andinos IFEA (2005; Lima, Perú). E-mail: estuki@gmail.com

${ }^{* * *}$ Departamento de Paleontología de Vertebrados. Museo de Historia Natural de la Universidad de San Marcos (Lima, Perú). E-mail: mariourbina01@hotmail.com 


\title{
Le registre de Pelagornithidae (Oiseaux : Pelecaniformes) et l'avifaune néogène du Sud-est Pacifique
}

\section{Résumé}

Cet article examine le registre néogène de la famille éteinte Pelagornithidae des Formations Pisco (Pérou) et Bahía Inglesa (Chili) sur la côte pacifique de I'Amérique du Sud. De nouveaux spécimens appartenant au genre Pelagornis des niveaux Sacaco et Aguada de Lomas de la Formation Pisco et du niveau phosphatique de la Formation Bahía Inglesa sont signalés. De même, on présente des éléments crâniens et postcrâniens d'un genre indéterminé du niveau Montemar de la Formation Pisco et de la base de cette dernière, à la limite du niveau Cerro La Bruja et de la Formation Chilcatay. On compare le présent registre avec les connaissances précédentes pour la famille dans l'hémisphère sud. On discute la problématique taxonomique du groupe et la bonne assignation des éléments fossiles à la famille. Finalement, la connaissance actuelle de l'avifaune néogène dans le secteur d'étude est commentée.

Mots clés : Pelagornithidae, Formation Pisco, Formation Bahia Inglesa, néogène

\section{The record of Pelagorninthidae (Aves: Pelecaniformes) and the Neogene avifauna of the southeast Pacific}

\begin{abstract}
The Neogene record of the extinct family Pelagornithidae is examined in the Pisco Formation (Peru) and Bahia Inglesa (Chile) Formations along the Pacific coast of South America. New specimens belonging to the genus Pelagornis are reported; these come from the Sacaco and Aguada de Lomas levels of the Pisco Formation and from the phosphatic level of the Bahia Inglesa Formation. In addition new cranial and postcranial elements of uncertain genus are discussed from the Montemar level of the Pisco Formation and the base of the same Formation at the limit of the Cerro la Bruja level and the Chilcatay Formation. The present record is compared with previously known antecedents for the family in the southern hemisphere. The taxonomic problems of the group and the correct assignment of fossil elements to the family are discussed. The current knowledge of the Neogene avian record with in the study area is commented upon.
\end{abstract}

Key words: Pelagornithidae, Pisco Formation, Bahia Inglesa Formation, Neogene

\section{INTRODUCCIÓN}

La familia Pelagornithidae (Furbringer, 1888) es un grupo extinto de aves marinas, que se desarrolló del Paleoceno tardío al Plioceno, y se consideran claramente cosmopolitas (Olson, 1985). Su rasgo más conspicuo es la existencia de numerosas proyecciones óseas a modo de dientes a lo largo de las tomias. Alcanzaban grandes envergaduras y poseían una extrema pneumatización en sus huesos, lo que dificulta su buena preservación. Los ejemplares más completos se han conservado de modo bidimensional, dificultando su análisis. Igualmente, la taxonomía del grupo es muy incierta, considerándose por lo general una única familia en el suborden Odontopterygia (Spulski, 1910), dentro del orden Pelecaniformes (Olson, 1985); aunque recientemente se ha propuesto una mayor afinidad con los Anseriformes (Bourdon, 2005). 
El registro de la familia en el hemisferio sur está restringido a cuatro localidades. En el Atlántico sudeste se conocen restos de dos especies de afinidad desconocida asignadas al Eoceno de la Isla Seymour, Antártida (Tonni \& Cione, 1978; Tonni, 1980; Tonni \& Tambussi, 1985). En el Pacífico sudoeste existen tres registros procedentes de Nueva Zelanda: Pelagornis miocaenus (Lartet, 1857) del Mioceno de Canterbury (Scarlett, 1972; Harrison \& Walker, 1976), Pseudodontornis stirtoni (Howard \& Warter, 1969) del Plioceno de Waitotaran y elementos de una especie indeterminada del Plioceno de Hawera y Canterbury (Mc Kee, 1985; Fordyce, 1991). En el Pacífico sudeste se conocen dos localidades neógenas en la costa de América del Sur que corresponden a la Formación Pisco (centro sur del Perú) y la Formación Bahía Inglesa (norte de Chile) (Chávez \& Stucchi, 2002). Solo recientemente se ha descrito la presencia de la familia en el extremo norte de América del Sur, en el Mioceno de Venezuela (Rincón \& Stucchi, 2005).

En el Perú, los primeros reportes de la familia Pelagornithidae son mencionados por Muizon \& DeVries (1985) sobre la base de materiales procedentes del Mioceno de la Formación Pisco. Posteriormente, dichos materiales son asignados como Pelagornis sp. por Cheneval (1993). Corresponden a un extremo proximal de húmero derecho y una epífisis proximal de un metacarpo, los cuales se encuentran depositados en el Museo Nacional de Historia Natural de París (Francia).

Los primeros restos asignables a esta familia en Chile corresponden a la primera falange alar del segundo dígito izquierdo y dos tarsometatarsos derechos (Walsh, 2000; Walsh \& Hume, 2001), ambos procedentes de la Formación Bahía Inglesa. Adicionalmente, se ha mencionado un posible coracoide derecho atribuido tentativamente a la familia (Walsh, 2000). Los especímenes mencionados se encuentran depositados en la colección de la Universidad de Portsmouth (Inglaterra).

\section{MATERIAL Y MÉTODO}

El objetivo del presente trabajo es estudiar el material atribuido a la familia Pelagornithidae depositado en la colección del Departamento de Paleontología de Vertebrados del Museo de Historia Natural de la Universidad Nacional Mayor de San Marcos (UMSM) — Lima, Perú—y en el Museo Paleontológico de Caldera (MPC) —Atacama, Chile—. No fue posible la comparación directa con elementos depositados en otras colecciones. Parte de los presentes materiales han sido reportados previamente por los autores (Chávez, 2001; 2005a; Chávez \& Stucchi, 2002). Se adopta el criterio sistemático de Olson (1985). Se sigue la nomenclatura osteológica en español equivalente a la presentada por Howard (1980) y se expresan las medidas en milímetros, tomadas con un calibre Vernier de 0,01 mm de incremento. Las edades asignadas para los niveles de la Formación Pisco, está basadas en Muizon \& DeVries (1985) y Marocco \& Muizon (1988). Las edades asignadas para la Formación Bahía Inglesa, están basadas en Achurra (2004).

\section{GEOLOGÍA Y FAUNA DESCRITA}

La Formación Pisco corresponde a la unidad más rica en vertebrados marinos del Neógeno sudamericano. Aflora en la costa sur del Perú desde Pisco y hasta el norte de Yauca, extendiéndose $50 \mathrm{~km}$ tierra adentro en Nazca e Ica. Está representada por diatomitas y areniscas con conglomerados. Muizon \& DeVries (1985) establecen siete localidades fosilíferas donde se ha establecido niveles específicos de la Formación, incluyendo Sacaco y Sacaco Sur (Plioceno temprano), Montemar (Mioceno tardío-Plioceno temprano), Aguada de Lomas y El Jahuay (Mioceno tardío) y Cerro La Bruja (Mioceno medio); además, en los últimos años se han identificado al menos dos nuevas localidades: Lomas y Yauca con edades aproximadas a 10 y 2 millones de años (Ma) respectivamente (Stucchi, 2003). 
Martín Chávez, Marcelo Stucchi, Mario Urbina

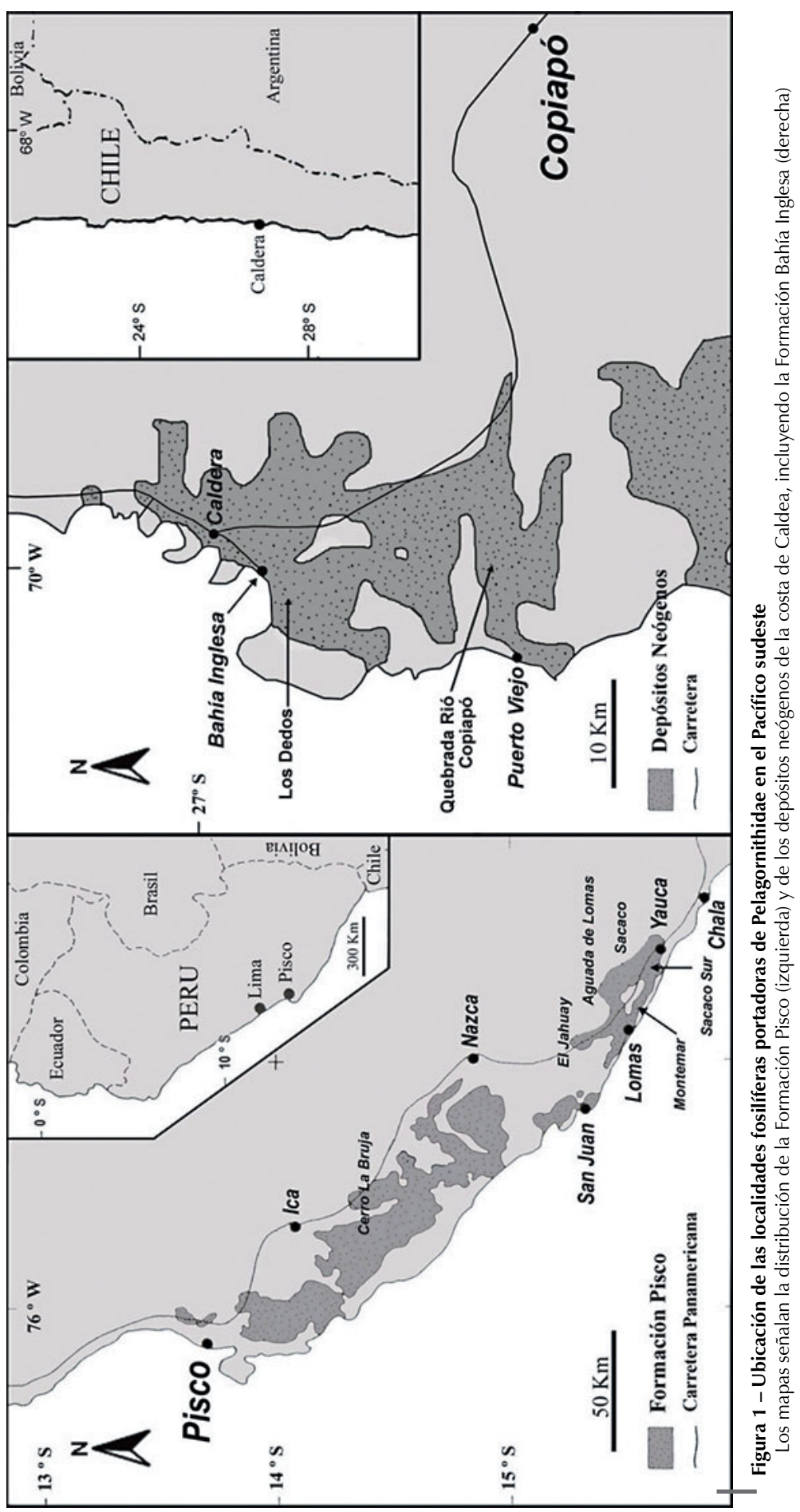


Existe un amplio rango de condiciones de depositación a lo largo de la columna, incluyendo playas agitadas, poco agitadas y arrecifes que han influido profundamente en las condiciones de conservación de los vertebrados de cada localidad (Marocco \& Muizon 1988). Se han sugerido condiciones de playas poco agitadas para las localidades Sacaco, Montemar y Cerro La Bruja; mientras que la localidad de Lomas correspondería a playas agitadas.

El estudio de los vertebrados en la Formación ha mostrado la existencia de numerosos mamíferos marinos (Muizon, 1981; Muizon \& McDonald, 1995; Muizon et al., 2002), junto con peces, reptiles y aves (Muizon \& DeVries, 1985; Stucchi, 2002; 2003). En el caso de las aves se conoce un total de 25 registros en 11 familias: Procellariidae (Boie, 1826); Diomedeidae (Gray, 1840); Spheniscidae (Bonaparte, 1831); Laridae (Rafinesque, 1815); Scolopacidae (Rafinesque, 1815); Vulturidae (Illiger, 1811); Ciconiidae (Gray, 1840); Phalacrocoracidae (Bonaparte, 1853); Sulidae (Reichenbach, 1849); Pelecanidae (Sharpe, 1891) y Pelagornithidae (Fürbringer, 1888) (Stucchi \& Urbina, 2005a).

En Chile, la localidad de Los Dedos $\left(27^{\circ} 00^{\prime} \mathrm{S}, 70^{\circ} 45^{\prime} \mathrm{W}\right.$ a $28^{\circ} 00^{\prime} \mathrm{S}, 71^{\circ} 00^{\prime} \mathrm{W}$ ) en las inmediaciones de la mina La Fosforita, en las proximidades de Bahía Inglesa (provincia de Copiapó, región de Atacama), es el afloramiento más importante de la Formación Bahía Inglesa y la más rica en aves fósiles de Chile (Chávez, 2005b). Esta unidad está representada por coquinas, areniscas y fangolitas, intercaladas con fosforitas y cenizas retrabajadas (Marchant et al., 2000). Los sedimentos fosfatados son particularmente ricos en fósiles de vertebrados marinos, cuyos huesos se acumulan localmente formando una cama de huesos (bonebed) (Walsh \& Hume, 2001). Estudios micropaleontológicos asignan una edad Mioceno medio a Plioceno Inferior para la unidad, sugiriendo ambientes marinos sublitorales a neríticos, junto con fuertes fluctuaciones en las condiciones climáticas con aguas subantárticas a cálidas (Marchant et al., 2000). Recientemente, la edad del techo de la Formación ha sido extendida hasta el Plioceno tardío (Achurra, 2004).

Los estudios paleontológicos en la Formación han revelado una gran variedad de vertebrados (Suárez \& Marquardt, 2003; Suárez et al., 2002) e invertebrados (Guzmán et al., 2000). En el caso de las aves, se conoce un total de 16 registros en seis familias: Procellariidae, Diomedeidae, Spheniscidae, Phalacrocoracidae, Sulidae y Pelagornithidae (Chávez, 2005b). Es oportuno mencionar que dadas las condiciones de depositación observadas en esta unidad, es usual hallar los especímenes inmersos en la matriz fosfática, lo cual dificulta la limpieza y preparación de las piezas dada su extrema dureza. En algunos de los especímenes aquí descritos es posible hallar fragmentos de fauna directamente asociada en la matriz. En el espécimen MPC 1001, se distinguen fragmentos óseos de mamíferos marinos, mientras que en MPC 1003 se observa un diente de Isurus oxyrhynchus. Un tratamiento más extenso de la geología de ambas áreas puede hallarse en Muizon \& DeVries (1985), Marocco \& Muizon (1988) y Achurra (2004).

\section{RESULTADOS}

\section{1. Sistemática paleontológica}

Orden PELECANIFORMES SHARPE, 1891

Suborden ODONTOPTERYGIA SPULSKI, 1910

Familia PELAGORNITIDAE FURBRINGER, 1888

Género PELAGORNIS LARTET, 1857

Especie tipo Pelagornis miocaenus LARTET, 1857 


\section{1. 1. Distribución estratigráfica y geográfica}

Mioceno medio; de la Molasse Coquillière marine, Armagnac, Francia; material referido en las Molasse de Léognan, Deognan, Francia (Mioceno medio); y en Waiauan, Canterbury, Nueva Zelanda (Mioceno medio-tardío) (Harrison \& Walker, 1976).

\section{1. 2. Diagnosis}

Húmero muy grande y delgado con eje recto. Extremo proximal estrecho y anteroposteriormente aplanado. La tuberosidad externa se extiende próximalmente más allá de la cabeza proximal, y se encuentra anconalmente desviada. Cabeza proximal pequeña y palmarmente inflada. El surco ligamental está bien definido. Fosa capital muy ancha y baja. La cavidad anconal es profunda y expandida desde la tuberosidad externa, formando junto con las otras socavaciones de la cabeza proximal una cadena distal, con la curvatura interna marcada, sobre la superficie anconal. La tuberosidad interna es grande y anconalmente prominente. No posee foramen neumático. La superficie bicipital es estrecha y está distalmente elongada, con una pequeña protuberancia redondeada en el extremo distal. La marca del $M$. brachialis anticus es muy alongada y pobremente definida. La prominencia ectepicondilar es grande. Cicatriz de anclaje profunda y anconal al entepicóndilo. La fosa olecranal es muy pequeña (según Harrison \& Walker, 1976).

\section{2. Pelagornis sp. (fig. 2)}

\section{2. 1. Material referido}

MUSM 209, húmero izquierdo con eje fragmentado y extremos conservados; MUSM 265, húmero derecho con eje fragmentado y extremo proximal conservado. Colectados por Mario Urbina en 1998. MPC 1000, extremo proximal de húmero derecho en matriz, carente de superficie palmar del eje. Colectado por Ricardo Chávez en 1999.

\section{2. 2. Procedencia geográfica}

MUSM 209, localidad fosilífera de Sacaco, departamento de Arequipa, Perú. MUSM 265, localidad fosilífera de Lomas, departamento de Arequipa, Perú. MPC 1000, localidad fosilífera de Los Dedos, Mina La Fosforita, Bahía Inglesa, región de Atacama (Chile).

\section{2. 3. Procedencia estratigráfica}

MUSM 209, nivel Sacaco de la Formación Pisco, Plioceno temprano (3,9-3 Ma). MUSM 265, localidad de Lomas de la Formación Pisco, Mioceno tardío (ca. 10 Ma). MPC 1000, nivel fosfático (bonebed) de la Formación Bahía Inglesa (Mioceno medio).

\section{2. 4. Descripción}

Húmero muy grande y delgado. Eje ligeramente curvado en sentido medial, acentuado en MUSM 209 por efecto de la compresión. Extremo proximal estrecho de bordes triangulares y faceta superior suavemente trilobulada (secuencia tuberosidad externa — cabeza — tuberosidad interna). Anteroposteriormente aplanado. La tuberosidad externa es amplia y anconalmente prominente. Su tamaño es menor al de la cabeza proximal, la cual es grande y palmarmente inflada. Palmarmente, el surco ligamental está bien definido, siendo más ancho al nivel de la tuberosidad interna que de la cabeza. Surco capital bajo y poco definido. Cavidad anconal baja, limitada proximalmente por la tuberosidad externa, la cabeza proximal y la tuberosidad interna, iniciándose bajo la concavidad formada por la tuberosidad externa y seguida por el margen convexo de la cabeza. La faceta interna y la superficie anconal de la tuberosidad interna están 
c.

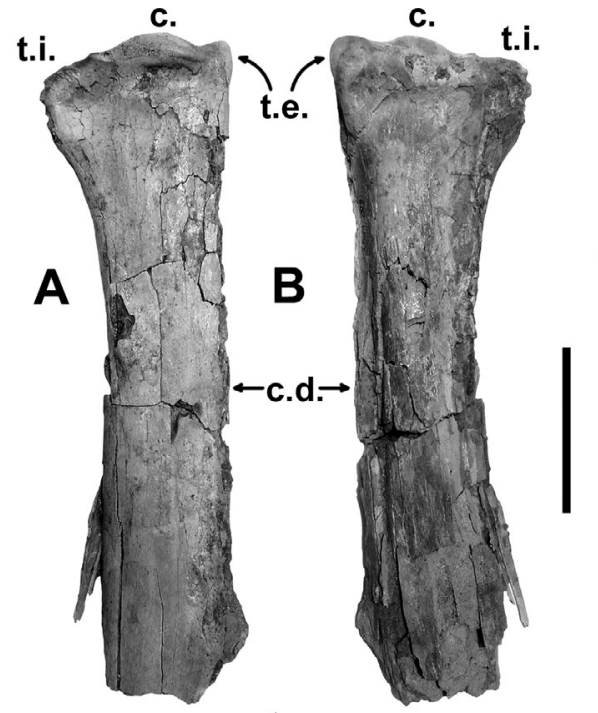

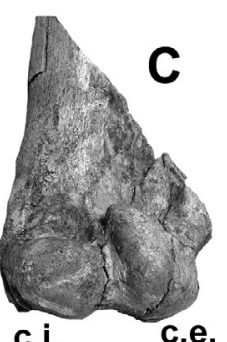

c.i. c.e.

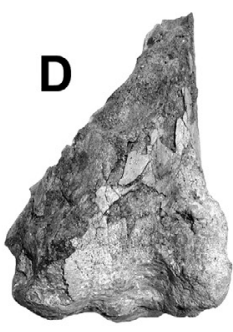

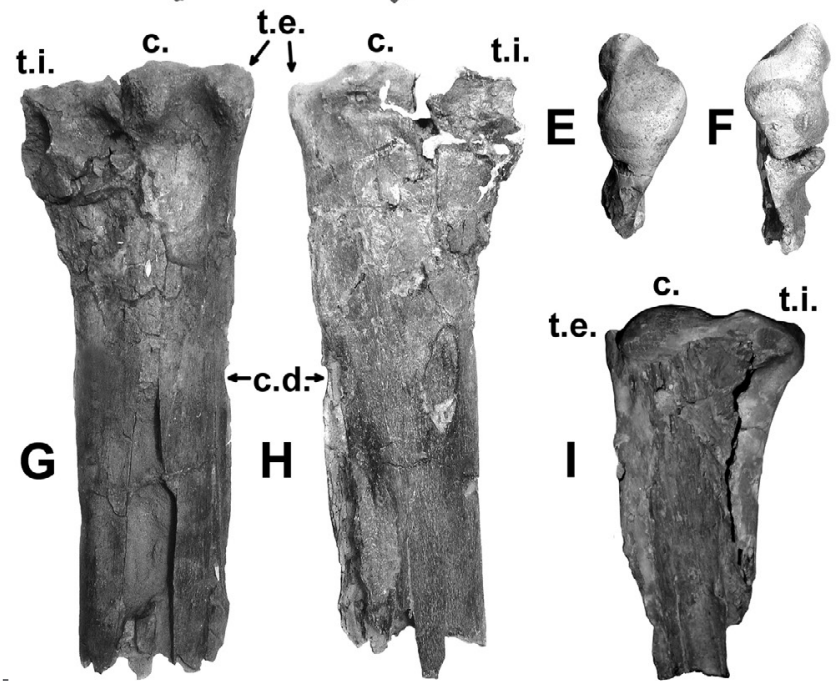

Figura 2 - Pelagornis sp. MUSM 209, húmero izquierdo -extremo proximal-

A. palmar

B. anconal

C. palmar

D. anconal. MUSM 666, extremo proximal de húmero derecho

E. proximal (extremo distal)

F. proximal

G. anconal

H. palmar, MPC 1 000, extremo proximal de húmero derecho

I. palmar

Abreviaturas: c. cabeza proximal; c.d. cresta deltoides; c.e. cóndilo externo; c. i. cóndilo interno; t.e. tuberosidad externa; t.i. tuberosidad interna. Escala: $5 \mathrm{~cm}$ 
erosionadas en el espécimen MUSM 209. El área de la tuberosidad interna y su superficie anconal y palmar están erosionados y fragmentados en MUSM 265. Base de la tuberosidad interna amplia y expandida, suavemente prominente en sentido anconal en MUSM 209. Tuberosidad interna grande, anconalmente prominente y de facetas rectas en MPC 1000. Fosa pneumática ausente. Superficie bicipital amplia próximalmente y angostándose progresivamente en dirección distal, con prominencia redondeada hacia el extremo distal. La superficie bicipital se ha perdido en el espécimen MPC 1000. La cresta deltoides está desgastada en los especímenes MUSM 209, MUSM 265 y MPC 1000, observándose una base delgada, baja y de borde recto. La superficie interna de la cara anconal se observa fuertemente pneumatizada y vascularizada en MPC 1000. Extremo distal estrecho conservado solo en MUSM 209. Marca del brachialis anticus en la depresión braquial, elongada y poco definida. Prominencia ectepicondilar grande. Cóndilos distales prominentes y bien definidos. La superficie intercondilar es regular. Ectepicóndilo grande, de superficie redondeada. Fosa olecranal pequeña. Marca para el ligamento articular anterior muy reducida. El área entre ésta y el cóndilo interno está fuertemente deprimida —ver las medidas en el cuadro 1 -

\section{2. 5. Comentarios}

A pesar de provenir de diferentes niveles de la Formación Pisco, ambos materiales son muy semejantes existiendo solo ligeras diferencias en el tamaño. MUSM 209 y 265 presentan medidas mayores que las descritas para Pelagornis miocaenus, mientras que MPC 1000 es intermedio entre esta especies y MUSM 209 y 265 (ver cuadro 1). No se reconocen diferencias significativas respecto a la diagnosis ofrecida (Harrison \& Walker, 1976).

\section{Cuadro 1 - Medidas de los húmeros}

Se presentan además las medidas ofrecidas por Harrison \& Walker (1976) para Pelagornis miocaenus (medidas expresadas en milímetros)

\begin{tabular}{|l|c|c|c|c|c|}
\hline & $\begin{array}{c}\text { MUSM } \\
\mathbf{2 0 9}\end{array}$ & $\begin{array}{c}\text { MUSM } \\
\mathbf{2 6 5}\end{array}$ & $\begin{array}{c}\text { MUSM } \\
\mathbf{6 6 6}\end{array}$ & $\begin{array}{c}\text { MPC } \\
\mathbf{1 0 0 0}\end{array}$ & $\begin{array}{c}\text { Pelagonis } \\
\text { miocaenus }\end{array}$ \\
\hline Ancho máximo interno-externo & 70,1 & 70,0 & 60,5 & 65,3 & 61,5 \\
\hline Ancho máximo palmar-anconal & 30,4 & 26,6 & 19,4 & - & - \\
\hline $\begin{array}{l}\text { Grosor anteroposterior de } \\
\text { la cabeza }\end{array}$ & 26,9 & 28,7 & 19,3 & - & 21,2 \\
\hline Ancho anconal de la cabeza & - & 25,8 & 17,4 & - & - \\
\hline Ancho palmar de la cabeza & 34,6 & 26,2 & - & 28,1 & - \\
\hline $\begin{array}{l}\text { Distancia anconal entre la } \\
\text { tuberosidad externa y la cabeza }\end{array}$ & 42,7 & 47,5 & 41,1 & 36,1 & - \\
\hline $\begin{array}{l}\text { Ancho proximal del Lassitum } \\
\text { dorsi posterioris }\end{array}$ & 23,5 & 20,1 & - & - & - \\
\hline $\begin{array}{l}\text { Ancho anteroposterior del } \\
\text { condilo interno }\end{array}$ & 53,3 & - & - & - & 37,6 \\
\hline $\begin{array}{l}\text { Ancho anteroposterior del } \\
\text { condilo externo }\end{array}$ & 18,8 & 25,1 & - & - & 32,5 \\
\hline
\end{tabular}




\section{3. Pelagornithidae indet. cf. Pelagornis (figs. 3-4)}

\section{3. 1. Material referido}

MPC 1001, porción anterior del rostro incompleto en matriz; MPC 1002, fragmento posterior del premaxilar derecho en matriz; ambos asociados. MPC 1003, porción superior del neurocráneo en matriz; MPC 1004, extremo proximal de ulna izquierda; MPC 1005, MPC 1006, vértebras cervicales aisladas. Colectados por Martín Chávez (MPC 1001, 1002, 1006), Fernando Suárez (MPC 1003) y Manuel Flores (MPC 1004, 1005) entre 2000 y 2002.

\section{3. 2. Procedencia geográfica}

MPC 1001, 1002, 1003, 1004, 1005; localidad fosilífera de Los Dedos, Mina La Fosforita, Bahía Inglesa, región de Atacama, Chile. MPC 1006; quebrada del río Copiapó, interior de Puerto Viejo, región de Atacama, Chile.

\section{3. 3. Procedencia estatigráfica}

MPC 1001, 1002, 1003, 1004, 1005; nivel fosfático (bonebed) de la Formación Bahía Inglesa, Mioceno medio. MPC 1006; colectado como rodado, posiblemente nivel de areniscas, Mioceno medio (i?).

\section{3. 4. Descripción}

MPC 1001, rostrum bajo y ancho, de corte transversal triangular y culmen fuertemente marcado. Se ensancha hacia el extremo posterior y se halla levemente curvado ventralmente. Los surcos laterales están sumamente marcados, paralelos al culmen y convergentes respecto a las tomias en el extremo anterior. En la parte anterior del fragmento el surco se encuentra en el cuarto inferior de la distancia entre el culmen y la tomia. La región palatar no es visible por el sedimento. No existe expansión palatar del rostro en la sección transversal. Las proyecciones tomiales son verticales, aplanadas e irregulares en su altura. Se observan cinco proyecciones principales, intercaladas por tres accesorias de tamaño considerablemente menos. No se distingue un patrón en las proyecciones. Su altura y separación aparecen en el cuadro 2 . Una rotura en la parte posterior de la tomia derecha muestra claramente la neumatización extrema del rostro y los finos puntales de las paredes óseas.

MPC 1002, fragmento posterior de premaxilar derecho. Presenta un surco lateral profundo que formando una $\langle\vee »$ invertida y ensanchada
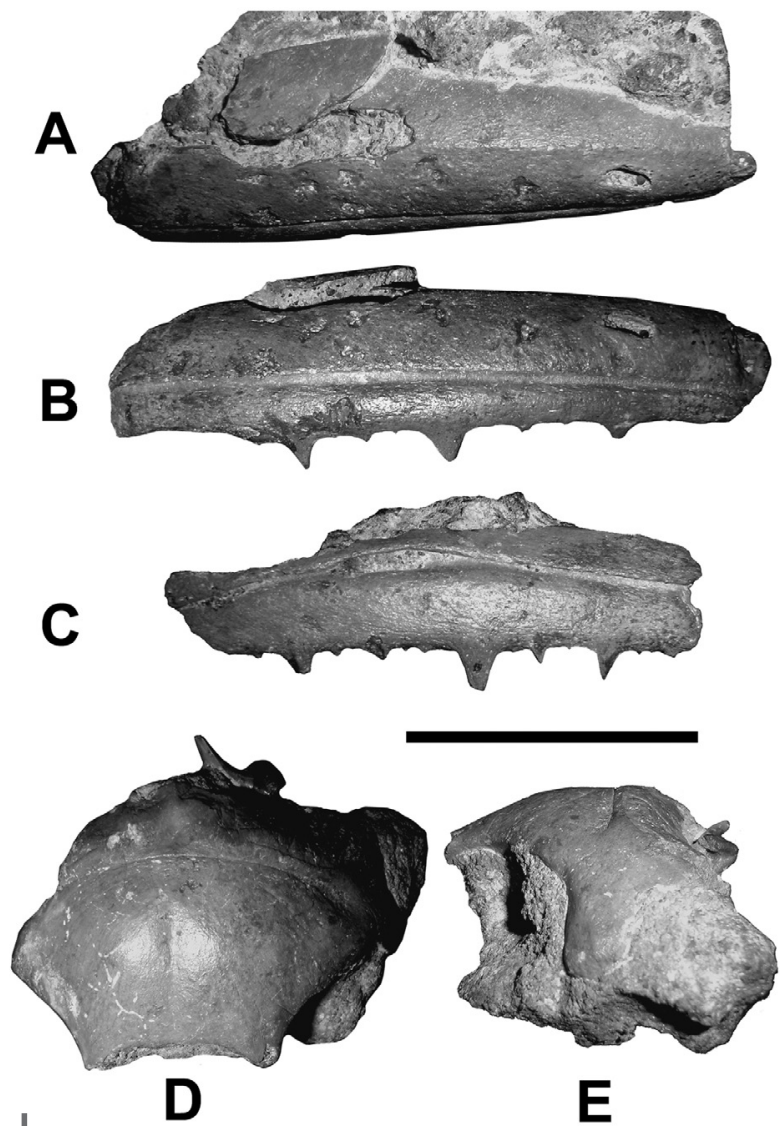

Figura 3 - Pelagornithidae indet. cf. Pelagornis MPC 1001, porción anterior del rostro.

A. dorsal; B. lateral derecho. MPC 1002, fragmento posterior del premaxilar derecho; C. lateral derecho. MPC 1003, región frontal parcial; D. dorsal; E. lateral izquierdo. Escala $5 \mathrm{~cm}$ 

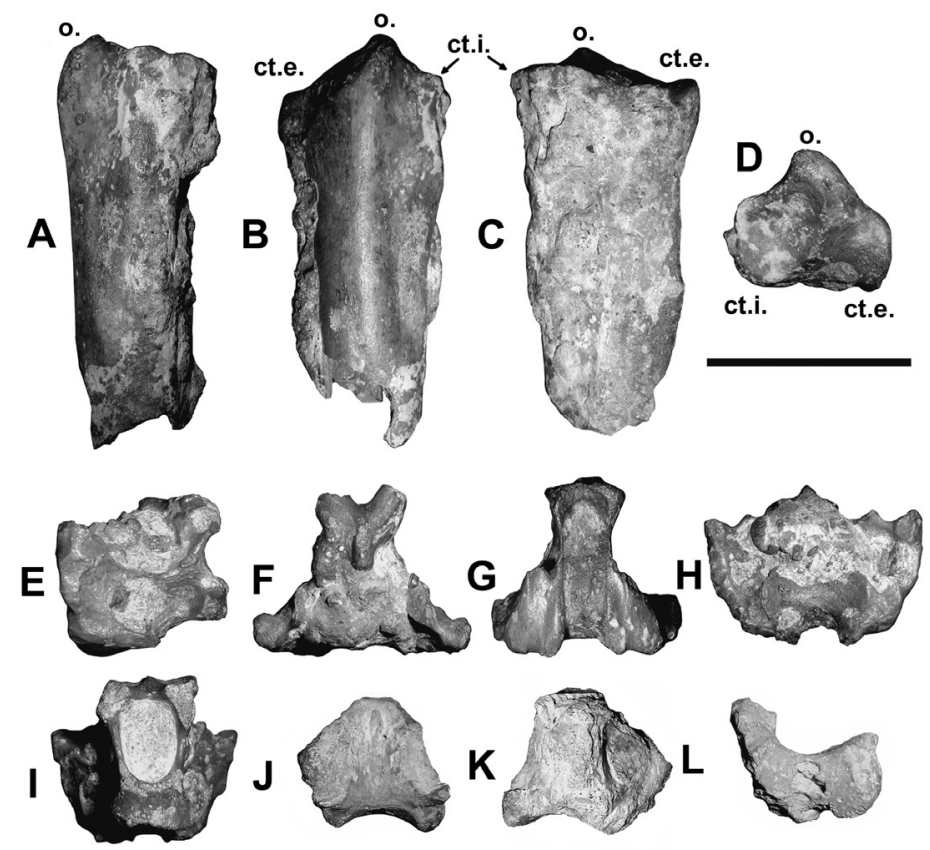

Figura 4 - Pelagornithidae indet. cf. Pelagornis MPC 1004, extremo proximal de ulna izquierda

A. interno; B. anconal; C. palmar; D. proximal. MPC 1005, vértebra cervical posterior; E. lateral izquierdo; F. dorsal; G. ventral; H. craneal; I. caudal. MPC 1006, cuerpo parcial de vértebra cervical posterior; J. dorsal; K. ventral; L. craneal. Abreviaturas: ct.e. cótilo externo; ct.i. cótilo interno; o. olécranon. Escala $5 \mathrm{~cm}$

en su vértice hacia la región posterior. Inmediatamente encima del vértice se observa una leve depresión. Las proyecciones tomiales se asemejan a las del rostro MPC 1001, presentando también las pequeñas proyecciones accesorias, observándose un total de trece proyecciones, de las cuales ocho son claramente visibles. No se distingue un patrón en las proyecciones. Su altura y separación aparecen en el cuadro 2. Las paredes óseas, a su vez, se encuentran vascularizadas.

MPC 1003, frontales posteriormente comprimidos y redondeados, con suave depresión mediodorsal. Sutura frontoparietal conspicua y adelantada, a la altura de los procesos postorbitales, convexa en sentido caudal y lateralmente deprimida. Proceso postorbital corto, amplio y marcado. Reborde supraorbitario liso.

MPC 1004, extremo de ulna muy grande. Superficie palmar del eje aplanada y parcialmente cubierta por sedimentos. Impresión del músculo brachialis anticus proximalmente profunda. Línea intermuscular fuertemente marcada e internamente desviada. Superficie articular con margen palmar cóncavo y margen anconal convexo. Cótilo interno en forma de «D», mayor al cótilo externo, con margen interno ligeramente cóncavo y margen palmar marcadamente convexo. Cótilo externo lateralmente prominente con margen externo suavemente convexo. Área intercotilar palmarmente comprimida y anconalmente expandida. Prominencia para el ligamento anterior articular muy expandida y lateralizada. La impresión bicipital y la depresión radial proximal no son visibles por el sedimento. Olécranon marcado, anconalmente prominente y con dirección interna. En vista anconal, eje olecranal fuertemente marcado. Depresión humeoulnar amplia. Marca tricipital parcialmente cubierta por sedimento, sin embargo, se observa fuertemente marcada. 
El registro de Pelagornithidae (Aves: Pelecaniformes) y la avifauna neógena del Pacífico sudeste

Cuadro 2 - Medidas de las proyecciones tomiales (pseudodientes) de los especímenes aquí descritos La numeración de las proyecciones se ilustra en la figura 5. Medidas expresadas en milímetros

\begin{tabular}{|c|c|c|c|c|c|c|}
\hline \multirow{2}{*}{\multicolumn{2}{|c|}{ PSEUDODIENTES }} & \multirow{2}{*}{$\begin{array}{l}\text { MPC } \\
1001\end{array}$} & \multirow{2}{*}{$\begin{array}{l}\text { MPC } \\
1002\end{array}$} & \multicolumn{3}{|c|}{ MUSM 210A } \\
\hline & & & & A & B & $\mathrm{C}$ \\
\hline \multicolumn{2}{|c|}{$\begin{array}{l}\text { Largo total del } \\
\text { fragmento }\end{array}$} & 108,6 & 98,3 & 65,2 & 77,1 & 30,1 \\
\hline \multirow[b]{2}{*}{1} & Altura & 6,1 & 4,5 & 7,0 & 8,7 & 6,0 \\
\hline & $\begin{array}{l}\text { Ancho } \\
\text { base }\end{array}$ & 4,6 & 5,6 & 4,3 & 4,9 & 3,7 \\
\hline \multirow[b]{2}{*}{2} & Altura & 1,2 & 9,0 & 4,6 & - & - \\
\hline & $\begin{array}{l}\text { Ancho } \\
\text { base }\end{array}$ & 1,7 & 1,4 & 2,4 & 3,0 & - \\
\hline \multirow[b]{2}{*}{3} & Altura & 6,6 & 2,3 & 5,7 & 5,7 & - \\
\hline & $\begin{array}{l}\text { Ancho } \\
\text { base }\end{array}$ & 7,1 & 3,3 & 3,4 & 3,0 & - \\
\hline \multirow[b]{2}{*}{4} & Altura & 1,9 & 7,0 & - & - & - \\
\hline & $\begin{array}{l}\text { Ancho } \\
\text { base }\end{array}$ & 2,5 & 8,0 & - & - & - \\
\hline \multirow[b]{2}{*}{5} & Altura & 3,8 & 8,1 & - & - & - \\
\hline & $\begin{array}{l}\text { Ancho } \\
\text { base }\end{array}$ & 4,8 & 7,0 & - & - & - \\
\hline \multirow[b]{2}{*}{6} & Altura & - & 2,7 & - & - & - \\
\hline & $\begin{array}{l}\text { Ancho } \\
\text { base }\end{array}$ & - & 2,1 & - & - & - \\
\hline \multirow[b]{2}{*}{7} & Altura & - & 6,5 & - & - & - \\
\hline & $\begin{array}{l}\text { Ancho } \\
\text { base }\end{array}$ & - & 4,4 & - & - & - \\
\hline \multirow[b]{2}{*}{8} & Altura & - & - & - & - & - \\
\hline & $\begin{array}{l}\text { Ancho } \\
\text { base }\end{array}$ & - & 2,8 & - & - & - \\
\hline \multicolumn{2}{|c|}{ Distancia entre 1 y 2} & 7,0 & 3,7 & 16,5 & 17,3 & - \\
\hline \multicolumn{2}{|c|}{ Distancia entre 2 y 3} & 9,0 & 5,8 & 17,4 & 18,2 & - \\
\hline \multicolumn{2}{|c|}{ Distancia entre 3 y 4} & 8,2 & 5,7 & - & - & - \\
\hline \multicolumn{2}{|c|}{ Distancia entre 4 y 5} & 11,6 & 5,0 & - & - & - \\
\hline \multicolumn{2}{|c|}{ Distancia entre 5 y 6} & - & 6,4 & - & - & - \\
\hline \multicolumn{2}{|c|}{ Distancia entre 6 y 7} & - & 8,2 & - & - & - \\
\hline
\end{tabular}


Vértebras cervicales muy grandes. MPC 1005, centro heterocélico dorsoventralmente comprimido, fuertemente cóncavo ventralmente, con superficie articular anterior amplia y mayor que la superficie articular posterior. Canal neural rellenado por los sedimentos, dificultando su observación, particularmente en su vista anterior. Posteriormente se observa alto y en forma de óvalo. El arco neural se observa alto, estrecho y posteriorizado. Espina neural muy baja. Diapófisis altas y anteriormente dirigidas. Prezygapófisis amplia en la superficie dorsal de las diapófisis. Solo se conserva la base de las postzygapófisis, estrechas y cortas. Parapófisis bajas y redondeadas. Pleurapófisis posteriormente fragmentada, de superficies amplias y delgadas. MPC 1006, centro heterocélico dorsoventralmente comprimido, fuertemente vascularizado y carente de apófisis. Superficie dorsal cóncava. Superficie ventral parcialmente cubierta por sedimentos, observándose cóncava. Superficie articular anterior amplia y mayor que la superficie articular posterior.

\section{3. 5. Comentarios}

La naturaleza fragmentaria de los materiales hallados dificulta enormemente la verificación de las diagnosis conocidas para los géneros de la familia. Los materiales rostrales aquí descritos difieren de los descubiertos en el Perú, no sólo por el tamaño, sino que también en la morfología pseudodental. Las proyecciones son más numerosas y robustas que las observadas en MUSM 210A. Estos materiales han sido previamente asociados al género Pseudodontornis (Chávez \& Stucchi, 2002) con base en las características de las proyecciones tomiales y la conformación de la región palatar del rostro; no obstante, en el presente se ha preferido utilizar un criterio más conservador a la espera de nuevos materiales. Los elementos postcraneales son mayores a los descritos como Pelagornithidae indet., coincidiendo en el rango de tamaño esperado para el género Pelagornis. Se evita la asignación estricta de los materiales a dicho género, dado el estado fragmentario de los especímenes y la inexistencia de materiales diagnósticos directamente asociados.

\section{MPC 1002}

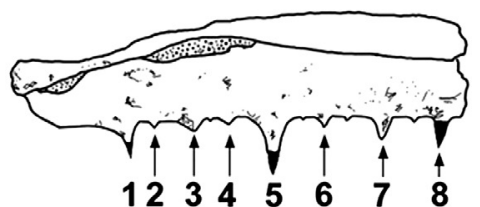

MPC 1001

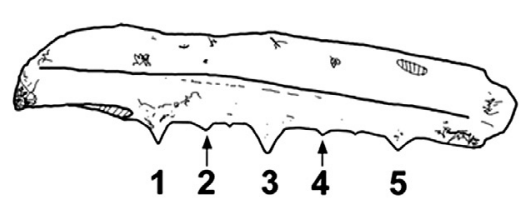

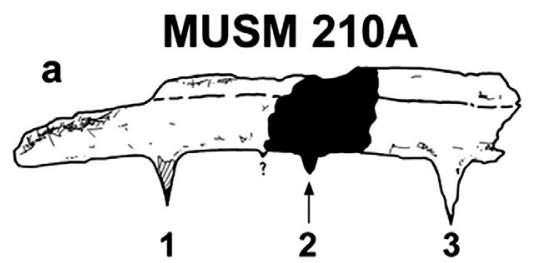
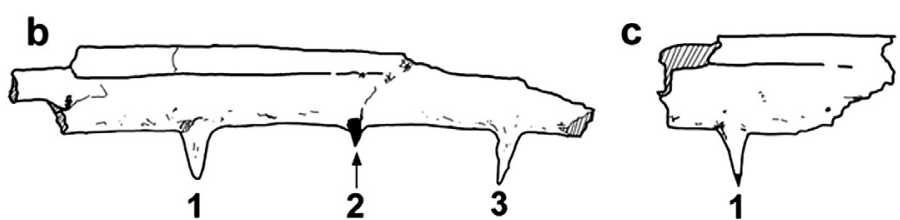

Figura 5 - Esquema de los elementos rostrales descritos en el presente texto Se numeran las proyecciones tomiales cuyas medidas se indican en el cuadro 2 


\section{4. Pelagornithidae gen. et sp. indet. (fig. 6)}

\section{4. 1. Material referido}

MUSM 210, fragmentos rostrales (MUSM 210A) y atlas asociados (MUSM 210B). Colectados por Mario Urbina en 1998. MUSM 666, extremo proximal (cabeza) de húmero derecho. MUSM 667, extremo proximal de ulna derecha y parte del eje. Colectados por Mario Urbina y Marcelo Stucchi en 2005.

\section{4. 2. Procedencia geográfica}

MUSM 210, localidad fosilífera de Montemar, departamento de Arequipa, Perú. MUSM 666 y 667, localidad fosilífera de Cerro La Bruja, departamento de Ica, Perú.

\section{4. 3. Procedencia estratigráfica}

MUSM 210, nivel Montemar de la Formación Pisco, Mioceno tardío-Plioceno temprano (6 Ma). MUSM 666 y 667, zona límite entre la Formación Pisco y la Formación Chilcatay, Mioceno medio (aproximadamente $15 \mathrm{Ma}$ ).

\section{4. 4. Descripción}

MUSM 210A, fragmentos rostrales muy delgados y bajos. No se conserva el culmen. La tomia está ligeramente curvada ventralmente. Los surcos laterales están bien definidos, paralelos a la tomia. No se conservan indicios de la región palatar. Las proyecciones tomiales son verticales, agudos, ligeramente cónicos, levemente irregulares en su altura y muy distanciados entre sí. La altura y separación de los pseudodientes aparecen en el cuadro 2. Las tomias están engrosadas respecto a la región más próxima al culmen.

MUSM 210B, Atlas grande. Solo se conserva la región articular con el cóndilo occipital y parte de los arcos que forman el canal neural. El orificio para el proceso odontoide del axis es abierto y estrecho. La superficie articular anterior es amplia, cóncava y profunda en forma de «U». La superficie articular posterior es amplia y profunda en forma de «U», más amplia que en la superficie anterior, con borde inferior convexo. No se aprecia la hipapófisis por encontrarse erosionada la parte ventral de la vértebra.

MUSM 666, húmero derecho de dimensiones menores a MUSM 209, MUSM 265 y ligeramente menores a MPC 1000. El extremo proximal es estrecho y fuertemente aplanado anteroposteriormente. La tuberosidad externa
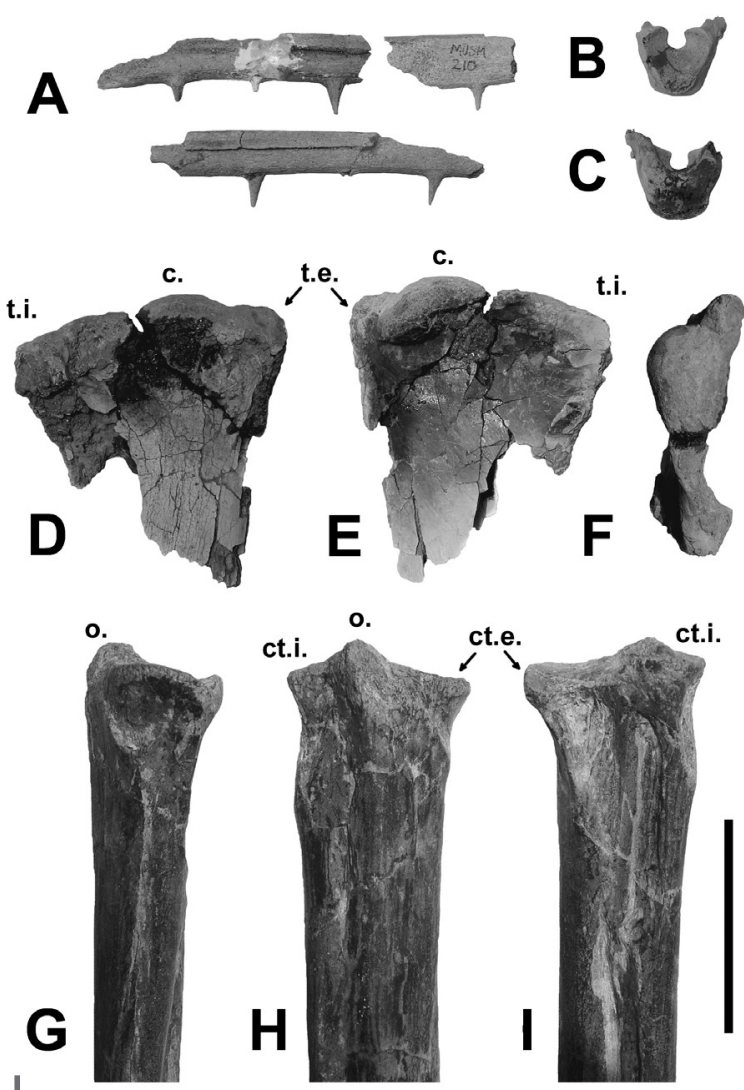

Figura 6 - Pelagornithidae indet. MUSM 210A, fragmentos rostrales A. lateral. MUSM 210B, atlas; B. caudal; C. craneal. MUSM 666, extremo proximal de húmero derecho; D. anconal; E. palmar; F. proximal. MUSM 667, extremo proximal de ulna derecha; G. externo; H. anconal; I. palmar. Abreviaturas: c. cabeza proximal; ct.e. cótilo externo; ct.i. cótilo interno; o. olécranon; t.e. tuberosidad externa; t.i. tuberosidad interna. Escala $5 \mathrm{~cm}$ 
es menor que la cabeza proximal y anconalmente desviada. La cabeza proximal pequeña y palmarmente inflada, es menor que en MUSM 209, MUSM 265 y MPC 1000. El surco ligamental es ancho y poco definido. La cavidad anconal es poco profunda y expandida desde la tuberosidad externa, formando junto con las otras depresiones de la cabeza proximal una cadena distal, con la curvatura interna marcada, sobre la superficie anconal. La tuberosidad interna está mal conservada, observándose grande y fuertemente prominente en sentido anconal. Fosa pneumática baja. La superficie bicipital es estrecha y distalmente elongada, con una pequeña protuberancia redondeada en el extremo distal (ver medidas en el cuadro 1).

MUSM 667, ulna derecha más grácil y de menor tamaño que MPC 1004, anteroposteriormente comprimida en la región media del eje. La superficie palmar del eje es aplanada. La impresión del músculo brachialis anticus está marcada y medial, la línea intermuscular suavemente marcada. El cótilo interno más profundo que el externo. El cótilo externo está fuertemente prominente palmarmente en dirección posterior. La prominencia para el ligamento anterior articular lateralizada es parcialmente conservada, la depresión radial proximal, fuertemente marcada. La depresión suave está debajo del cótilo interno. El olécranon está marcado, anconalmente más prominente que en MPC 1004 y con dirección interna. En vista anconal, el eje olecranal está marcado. La depresión humeroulnar es amplia.

\section{4. 5. Comentarios}

La separación y la forma de los dientes en MUSM 210A, es diferente de la observada en la mayoría de los materiales conocidos. El holotipo de Pseudodontornis longirostris (Lambrecht, 1930) presenta un patrón similar al observado en el material peruano, el que tras el hallazgo de nuevos especímenes se interpretó como ocasionado por la erosión de los dientes intermedios (Harrison \& Walker, 1976), algo que no parece poder verificarse en el presente material. La morfología del atlas MUSM 210B, presenta gran similitud con Osteodontornis Howard, 1957 (Howard \& White, 1962) de América del Norte. Se diferencia por presentar un orificio para el proceso odontoide abierto y una superficie articular posterior ligeramente más amplia. Se evita la asignación de MUSM 210 al género Osteodontornis por las diferencias en los elementos rostrales y la condición fragmentaria del espécimen. Los elementos apendiculares aquí incluidos son ligeramente más pequeños que los descritos como Pelagornis sp. La morfología general del húmero MUSM 666 concuerda con la diagnosis del género Pelagornis; no obstante la cabeza proximal es más pequeña y la tuberosidad interna es mucho más prominente en sentido anconal que en los especímenes aquí presentados. Se evita la asignación genérica fundamentalmente en virtud de la existencia de materiales de tamaño similar en los mismos niveles y el estado fragmentario del espécimen. La ulna MUSM 667 es menor que la MPC 1004, presentando además similitudes morfológicas generales. Sin embargo, la superficie palmar es más cóncava y el cótilo externo es más prominente al igual que el olécranon, pero menos que lo observado en especímenes referidos a los géneros Argillornis Owen, 1878 y Odontopteryx Owen, 1873 (Harrison \& Walker, 1976). Los elementos apendiculares aquí descritos sugieren la existencia de una segunda variedad de Pelagonithidae en la base de la Formación Pisco, de talla ligeramente menor a la observada en Pelagornis. No es posible realizar una asociación directa entre los materiales rostrales con los postcraneales, sin embargo son claramente distinguibles de los hallados en Chile hasta la fecha.

\section{DISCUSIÓN}

\section{1. Observaciones taxonómicas}

La familia Pelagornithidae constituye uno de los clados más misteriosos del Terciario. En la actualidad, se conoce un mínimo de 36 registros de estas aves en los depósitos terciarios de 
todos los continentes. Un listado reciente de los materiales asignados a la familia puede revisarse en Warheit (2002). Son numerosos los intentos por establecer la correcta taxonomía de la familia. Una de las más extensas corresponde al trabajo de Harrison \& Walker (1976), sin embargo, Olson (1985) desestima las conclusiones de este estudio, calificándolo de irreal. Es este mismo autor quien nos ofrece algunas de las aproximaciones más recientes a la taxonomía de la familia (Olson, 1985; Olson \& Rasmussen, 2001), sugiriendo la imposibilidad de establecer una correcta clasificación de los Pelagornithidae en base al material conocido, incluso a nivel genérico. Esto se debe a la ausencia de ejemplares completos, conociéndose muy pocos especímenes que incluyan elementos craneales y postcraneales asociados, imposibilitando la asignación genérica de dichos materiales. Una sugerencia planteada por Olson \& Rasmussen (2001) para la designación de los materiales hallados durante el Oligoceno tardío y el Neógeno en el Atlántico norte, es el uso del género más antiguo descrito en el área, Pelagornis, diferenciando especies solo en base a diferencias marcadas de tamaño. Una discusión sobre las limitaciones de la morfometría en la clasificación de aves fósiles es expuesta en Stucchi $(2002 ; 2003)$ y Urbina \& Stucchi (2005). Solo recientemente se han publicado estudios filogenéticos basados en especímenes asociados hallados en Marruecos (Paleoceno tardío-Eoceno temprano), los que revalidarían el uso del orden Odontopterygiformes para la familia (Bourdon, 2005).

Adicionalmente, las publicaciones existentes no aportan información detallada de las medidas de los especímenes, ni realizan las comparaciones necesarias para descartar la sinonímia de los taxones. Es en este sentido que el estudio de Harrison \& Walker (1976) adolece de criterios útiles para la diferenciación taxonómica, ofreciendo diagnosis basadas en caracteres ambiguos y no correlacionados entre taxones.

Solo se han descrito en detalle los húmeros de tres géneros de la familia Pelagornithidae: Osteodontornis, Argillornis y Pelagornis. En Osteodontornis el material referido consiste en un fragmento distal del húmero izquierdo, distorsionado y asociado al holotipo. La conservación de este material no permite establecer una correcta diagnosis para los elementos postcraneales, dificultando su comparación con los presentes especímenes. Sin embargo, se observa que en el espécimen MUSM 265 el proceso ectepicondilar se encuentra menos desarrollado que en el género Osteodontornis. Los fragmentos de húmeros referidos al género Argillornis, se encuentran fuertemente erosionados pero se distinguen por poseer un extremo proximal más redondeado y amplio que en los otros géneros. Sin duda, los elementos mejor conservados son los atribuidos al género Pelagornis. Los presentes materiales son claramente asignables a este género, difiriendo levemente de la especie tipo. Sin embargo, considerando el estado de conservación de los húmeros previamente descritos, es imposible determinar si la diagnosis del género Pelagornis aporta caracteres distintivos a nivel genérico o corresponden a caracteres comunes a la familia.

Respecto a otros elementos postcraneales, se han mencionado numerosos materiales atribuibles a la familia, he incluso a algunos géneros particulares. Existen también otros géneros establecidos con base en elementos postcraneales, como es el caso de Gigantornis (Andrew, 1916), cuyo holotipo consiste en un esternón incompleto. Muchos de los otros materiales postcraneales han sido asignados a taxones particulares, solo por correlación estratigráfica o del rango de tamaño (Lydekker, 1891; Harrison \& Walker, 1976; Gonzalez et al., 2002; 2004), ambos criterios ambiguos que no garantizan una directa relación cogenérica. Se reconoce sin embargo, que dadas las dificultades aquí planteadas, estos criterios artificiales parecen ser una solución transitoria para la catalogación de elementos aislados asociados a esta familia (Olson \& Rasmussen, 2001).

Por otra parte, se conocen numerosos materiales craneales de estas aves, sin embargo, son pocos los elementos bien conservados. Como consecuencia de esto, las diagnosis para estos elementos no han sido correctamente establecidas. En el caso de los géneros Pseudodontornis y Osteodontornis, que presentan los cráneos mejor conservados del Neógeno, las principales diferencias han sido establecidas en base a proporciones del pico respecto al neurocráneo (Harrison \& Walker, 1976); las que no pueden ser verificadas en especímenes fragmentados. Adicionalmente, el valor diagnóstico de caracteres tales como el número o forma de los dientes rostrales ha sido descartado, dada la alta variabilidad de tales rasgos incluso dentro de un mismo 
espécimen (Olson, 1985; Rincón \& Stucchi, 2005), invalidando alguno de los caracteres que separaban a ambos géneros. De modo que, con base en las actuales diagnosis, nos resulta imposible asignar los elementos craneales a un género en particular, a menos que se hallen en directa asociación con elementos postcraneales. Esto no ha ocurrido con los materiales aquí presentados, pues siempre se han hallado disgregados, con excepción de los materiales rostrales como se ha mencionado.

De esta forma, es imposible descartar la sinonimia entre taxones que han sido descritos en base a diferentes elementos esqueléticos, como es el caso de Pseudodontornis respecto a Pelagornis. Ambos géneros poseen rangos de tamaño similar, son sincrónicos y posiblemente simpátridos. En Nueva Zelanda, se han hallado elementos asociables a ambos géneros que proceden de localidades y edades diferentes (Fordyce, 1991). La presencia de al menos tres especies de Pelagornithidae en London Clay, Inglaterra (Harrison \& Walker, 1976; Olson \& Rasmussen, 2001), sugiere que estas aves podían vivir en simpatría. Algo similar ocurre con los materiales hallados en la Antártida (Tonni \& Tambussi, 1985) y en Carolina del Norte, EE.UU. (Olson \& Rasmussen, 2001). En todos estos casos existen marcadas diferencias de tamaño entre cada forma o claras diferencias morfológicas. En base a estos criterios, es posible distinguir la existencia de al menos dos variedades de Pelagornithidae en el Pacífico sudeste durante el Neógeno: Pelagornis sp. y Pelagornithidae indet.; distinguibles por diferencias morfológicas y de tamaño. En el caso de los materiales descritos como Pelagonithidae indet. cf. Pelagornis, no es posible una asignación estricta al género Pelagornis sólo con base a la coincidencia en el rango de tamaño, pudiendo o no corresponder a elementos congenéricos.

Los materiales previamente descritos por Cheneval (1993) como Pelagornis sp. de la Formación Pisco, consisten en un extremo proximal de humero procedente de la localidad El Jahuay (Mioceno tardío) y una epífisis proximal de un metacarpo de la localidad Sacaco Sur (Plioceno temprano). Con base a las ilustraciones publicadas, la identificación genérica parece justificarse para el espécimen de El Jahuay; sin embargo, dadas las problemáticas previamente comentadas, la identificación de la epífisis metacarpal debe considerarse como tentativa. En el caso de los especimenes descritos por Walsh \& Hume (2001) para la Formación Bahía Inglesa, estos coinciden en el rango de tamaño esperado para Pelagornis, pero por tratarse de elementos postcraneales inconexos se hallan en la situación descrita para Pelagornithidae indet. cf. Pelagornis.

En consideración del registro conocido para otras localidades en el hemisferio y en el continente, podemos decir que existen claras similitudes con la situación descrita para los registros neozelandeses (Fordyce, 1991). La presencia del género Pelagornis (Scarlett, 1972; Harrison \& Walker, 1976) en ambas áreas, sugiere cierta similitud en las variedades de aves pelágicas que pudieron habitar el Pacífico sur durante el Neógeno, la cual aun no ha sido evidenciada en otras aves, dado el escaso conocimiento de la avifauna marina neozelandesa durante el Mioceno (Fordyce, 1991). Los especímenes conocidos para el Paleógeno antártico son difícilmente contrastables con los aquí descritos, descartándose una correspondencia directa en el caso de los elementos rostrales con base en las fuertes diferencias de tamaño y la morfología de los mismos (Tonni, 1980). Finalmente, el material descrito para el Mioceno de Venezuela (Rincón \& Stucchi, 2005), presenta las mismas dificultades nomenclaturales que las abordadas por el presente trabajo, pudiendo o no tener relación con los especímenes descritos para la costa pacífica de América del Sur. De momento no se conocen elementos contrastables con los presentados para Venezuela, dentro de la presente área de estudio.

El uso de las especies descritas en base a materiales incompletos, debe considerarse como una alternativa provisoria de catalogación, a la espera de especímenes más completos, por lo que se recomienda realizar identificaciones solo a nivel familiar o genérico; así como la abstención de crear nuevas especies que entorpezcan aún más la revisión taxonómica, como ha sido sugerido por otros autores (Olson, 1985; Olson \& Rasmussen, 2001; Fordyce, 1991). El uso de estas especies en análisis cladísticos (Bourdon, 2004) resulta altamente especulativo, dadas las dificultades previamente descritas. 


\section{2. Avifauna del Pacífico sudeste durante el Neógeno tardío}

Los depósitos fosilíferos neógenos conocidos para la costa pacífica de América del Sur, nos permiten conocer con extraordinario detalle la paleofauna del Pacífico sudeste. La Formación Pisco es sin duda la más extensamente estudiada durante el siglo XX entregando importantes hallazgos en el estudio de los mamíferos marinos fósiles (Muizon, 1978; 1981; Muizon \& McDonald, 1995; Muizon et al., 2002); sin embargo, sólo recientemente la ornitofauna de la Formación ha sido correctamente reportada (Stucchi, 2002; 2003). Los materiales descubiertos en la Formación Bahía Inglesa han revelado también una inmensa riqueza y variedad de especies fósiles (Chávez, 2005a; Suárez et al., 2002; Suárez \& Marquardt, 2003; Acosta et al., 2002; Walsh \& Hume, 2001), sin embargo, muchas de ellas aún esperan su publicación formal. Los paralelos faunísticos entre ambas formaciones son impresionantes, observándose taxones prácticamente idénticos en ambas localidades (Chávez \& Stucchi, 2002; Chávez, 2005a; 2005b; Suárez \& Marquardt, 2003). Asimismo, otras formaciones de edades similares presentan registros faunísticos análogos, como es el caso de la Formación Coquimbo en Chile (Chávez, 2005c). En el caso particular de la ornitofauna podemos detallar la presencia de las siguientes familias:

\section{2. 1. Spheniscidae}

Los pingüinos fósiles son las aves más abundantes en las Formaciones marinas neógenas de Chile y Perú. Al menos pueden diferenciarse cuatro especies en la Formación Pisco (Stucchi \& Urbina, 2005b) y nueve especies en Bahía Inglesa, incluyendo las especies presentes en Perú (Chávez, 2005a; 2005b). Estas corresponden a un Sphenisciformes indet. de la parte basal de la Fm. Pisco ( $\sim 15 \mathrm{Ma}$ ) y dos formas extintas de Spheniscus: S. urbinai Stucchi, 2002 y S. megaramphus (Stucchi et al., 2003), del Mioceno tardío de Pisco; más una forma de menor tamaño y aún sin describir, posiblemente asociable al mismo género y de la misma antigüedad (Stucchi \& Urbina, 2005a). En Bahía Inglesa se ha reportado adicionalmente la existencia de los géneros argentinos Palaeospheniscus y Paraptenodytes (Fritis, 2001; Acosta et al., 2002; Acosta \& Canto, 2005), identificación no plenamente compartida por otros autores (Chávez, 2006; Chávez, en prensa); junto con una nueva especie conocida solo para dicha Formación del género Pygoscelis (AcostaHospitaleche et al., 2006). Existe una segunda especie de Pygoscelis en la Formación, de talla similar a la del actual género Aptenodytes (Walsh \& Suárez, 2006). Se ha sugerido la existencia de colonias en ambas formaciones, con base a la abundancia de material y la existencia de individuos jóvenes. Otras formaciones neógenas donde se ha reportado la presencia de pingüinos dentro del área de estudio, son la Formación Chilcatay (Mioceno temprano-medio) en el departamento de Ica, Perú, con el género Palaeospheniscus (Acosta \& Stucchi, 2005); la Formación Coquimbo (Mioceno medio-Plioceno temprano) en las regiones de Coquimbo y Atacama, Chile, con el género Spheniscus (Chávez, 2005c) y una forma afín a Paleospheniscinae (Tambussi et al., 2005) y la Formación La Portada (Plioceno tardío) en la región de Antofagasta, Chile, con la presencia Spheniscus chilensis (Emslie \& Guerra, 2003).

\section{2. 2. Procellariiformes (Diomedeidae y Procellaridae)}

Se conocen escasos restos representativos de estas aves en la Formación Pisco, a pesar de estar presentes en casi todos los niveles. Se conocen restos pertenecientes a la familia Procellaridae, designados como Fulmarus sp. (Cheneval, 1993) y Puffinini indet., junto con especímenes atribuidos a la familia Diomedeidae (Stucchi \& Urbina, 2005c). Existe una mayor diversidad en la Formación Bahía Inglesa, observándose al menos dos formas de Procellaridae y dos formas de Diomedeidae, destacando los géneros Diomedea y Pachyptila (Chávez, 2005b; Walsh \& Hume, 2001; Salaberry et al., 2007). Adicionalmente se ha reportado la presencia de Diomedeidae en la Formación Coquimbo (Chávez, 2005c). 


\section{2. 3. Phalacrocoracidae}

La Formación Pisco posee un amplio registro de esta familia observándose al menos dos formas de cormoranes fósiles, uno de dimensiones comparables con la especie más abundante del Pacífico sudeste actual, P. bougainvillii y presente solo a partir del Plioceno, y el otro de menor tamaño, conocido desde el Mioceno medio (Urbina \& Stucchi, 2005). En Chile son escasos los restos asociados a esta familia, designándose solo a nivel genérico como Phalacrocorax sp. del mismo modo que se designan las variedades identificadas en Pisco (Chávez, 2005b; Walsh \& Hume, 2001; Emslie \& Guerra, 2003). Es muy posible que las formas peruanas se correspondan con las chilenas, particularmente las de Bahía Inglesa de similar rango de tamaño; pero los escasos estudios realizados en el área y lo fragmentario de los materiales chilenos, ha impedido la comparación acabada de los materiales.

\section{2. 4. Sulidae}

Se ha descrito la presencia de al menos cuatro formas de piqueros en la Formación Pisco, correspondiendo a dos nuevas especies del género Sula -S. magna (Stucchi, 2003) y S. sulita (Stucchi, 2003), S. aff. variegata y Morus peruvianus (Stucchi, 2003)_; único registro formal de este género en América del Sur. Adicionalmente, se ha descrito el género Ramphastosula (Stucchi \& Urbina, 2004) para la Formación Pisco, un piquero especializado a partir de formas anteriores del género Sula. Las formas descubiertas en Bahía Inglesa han sido referidas al género Sula (Walsh \& Hume, 2001), observándose ejemplares que pueden concordar con las especies halladas en el Perú. Se ha sugerido también la presencia del género Morus en Bahía Inglesa (Walsh, comunicación personal). Adicionalmente se ha reportado la presencia de Sula sp. para la Formación Chilcatay (Mioceno temprano-medio) de Perú (Stucchi \& DeVries, 2003).

\section{2. 5. Pelecanidae}

El registro de esta familia en América del Sur es muy escaso, reportándose para el nivel Montemar de la Formación Pisco con base en un cuadrado aislado (Stucchi \& Urbina, 2005a). Están ausentes del registro fósil chileno.

\section{2. 6. Charadriiformes (Scolopacidae y Laridae)}

Solo en la Formación Pisco se conocen materiales referibles a este orden de aves marinas, con base en materiales altamente fragmentarios y reportados como Larus sp., cf. Limosa y Scolopacidae indet. (Stucchi \& Urbina, 2005a). Aún no se conocen materiales fósiles en Chile.

\section{2. 7. Vulturidae}

La Formación Pisco ha aportado algunos registros de fauna continental y litoral que amplían el rango de especies conocidas en el área. Uno de estos registros corresponde al cóndor Perugryps diazi (Stucchi \& Emslie, 2005) que es el primer registro de estas aves para el margen litoral de la costa pacífica de América del Sur.

\section{2. 8. Ciconiidae}

Se ha reportado esta familia para la Formación Pisco, mediante la identificación de un tarsometatarso fragmentario (Stucchi \& Urbina, 2005d).

\section{2. 9. Falconidae}

Existe solo un registro para la familia, Milvago sp., procedente de la Formación La Portada, Chile (Emslie \& Guerra, 2003). Corresponde al único registro de ave no estrictamente acuática en Chile continental. 


\section{CONCLUSIONES}

Todas las familias antes mencionadas se encuentran presentes en la actualidad en el mismo territorio, observándose una fuerte continuidad en la avifauna marina de la costa pacífica desde el Neógeno. La insuficiencia de información sobre las faunas marinas paleógenas de la costa pacífica, nos impide conocer a cabalidad la evolución temprana de estos ecosistemas y el origen exacto de la actual diversidad que al parecer ya se había establecido para el Neógeno tardío. Sólo recientemente se han presentado los primeros registros de Spheniscidae para el EocenoOligoceno del Perú (Stucchi \& Urbina, 2005a; 2005b; Acosta \& Stucchi, 2005). De todos los taxones mencionados solo Ramphastosula y Pelagornithidae se encuentran completamente extintos en la actualidad. Aún es escasa la información que tenemos del primer grupo, considerado como un grupo de piqueros especializado posiblemente en una dieta durofaga, cuya extinción podría relacionarse con los cambios faunísticos de fines del Neógeno.

El rol de los Pelagornithidae en los ecosistemas marinos no ha sido establecido con precisión, sugiriéndose una posición similar a la de los actuales Pelecanidae (Cheneval, 1993: 90) y Diomedeidae (Olson, 1985: 200), cuya extinción podría relacionarse con el reemplazo ecológico por parte de ambas familias durante el Plioceno. El registro de Pelagornis sp. en los niveles Sacaco (MUSM 209) y Sacaco Sur (Cheneval, 1993) de la Formación Pisco, confirma su presencia durante el Plioceno temprano en Sudamérica, constituyendo un aporte al escaso registro de la familia durante el Plioceno, previamente concentrado en Nueva Zelanda (Howard \& Warter, 1969; Mc Kee, 1985; Fordyce, 1991) y solo recientemente ampliado a Marruecos (Mourer-Chauviré \& Geraads, 2004). Estos registros dispersos muestran una distribución amplia del grupo aun durante el Neógeno terminal. Se ha mencionado una posible asociación entre la presencia de Pelagornithidae y familias de aves especializadas al buceo, Spheniscidae en el hemisferio sur y Plotopteridae en el hemisferio norte (González et al., 2002); que parece repetirse en los registros aquí expuestos.

Es claro que la diversidad de pingüinos en el área durante el Neógeno fue mayor que la actual, siendo un elemento dominante de la ornitofauna marina sudamericana. Igualmente, el número de especies registradas es mayor en la Formación Bahía Inglesa que incluye el género Pygoscelis, típico de zonas antárticas y subantárticas, lo que sugiere la presencia permanente de corrientes frías durante el neógeno en la costa Pacífica de América del Sur (Chávez, 2005b; Walsh, 2004; Acosta-Hospitaleche et al., 2006). El aumento de la diversidad en la Formación Bahía Inglesa se repite en el caso de los Procellariiformes que también se han asociado a corrientes frías. Esto sugeriría que las corrientes frías podrían haber sido más intensas hacia el sur. La relación inversa parece observarse en el caso de los Sulidae, mejor representados en Pisco y asociados a climas más cálidos.

La incidencia de aves continentales y los escasos registros de aves costeras en estas localidades responden principalmente a factores tafonómicos. En este sentido, las condiciones de depositación calmas y costeras descritas para la Formación Pisco (Marocco \& Muizon, 1988), resultan más apropiadas para la conservación de estas aves que las condiciones litorales de la Formación Bahía Inglesa (Chávez, 2005a). En consecuencia, los registros de aves continentales y costeras se concentran en la Formación Pisco, lo que no implica la inexistencia de tales animales en Bahía Inglesa. Las aves costeras no son un elemento dominante en el registro, sin embargo existe una correspondencia entre las familias identificadas y las que hoy habitan en la costa oeste de América del Sur (Pelecanidae y Charadriiformes). Los taxones continentales registrados pueden asociarse a hábitos oportunistas o carroñeros (Vulturidae, Ciconiidae y Falconidae). 


\section{Referencias citadas}

ACOSTA, C. \& CANTO, J., 2005 - Primer registro de cráneos asignados a Palaeospheniscus (Aves: Spheniscidae) procedentes de la Formación Bahía Inglesa (Mioceno mediotardío). Revista Chilena de Historia Natural, 78: 489-495; Chile.

ACOSTA, C., FRITIS, O., TAMBUSSI, C. \& QUINZIO, A., 2002 - Nuevos restos de pingüinos (Aves Spheniscidae) en la Formación Bahía Inglesa (Mioceno superior-Plioceno inferior) de Chile. In: Actas del Primer Congreso Latinoamericano de Paleontología de Vertebrados: 14; Santiago.

ACOSTA, C. \& STUCCHI, M., 2005 - Nuevos registros de Spheniscidae de la costa peruana. Revista Española de Paleontología, 20: 1-6; Valencia: Sociedad Española de Paleontología.

ACOSTA-HOSPITALECHE, C., CHÁVEZ, M. \& FRITIS, O., 2006 - Pingüinos fósiles (Pygoscelis calderensis nov. sp.) en la Formación Bahía Inglesa (Mioceno Medio- Plioceno). Revista Geológica de Chile, 33: 327-338; Santiago.

ACHURRA, L., 2004 - Cambios del nivel del mar y evolución tectónica de la cuenca Neógena de Caldera, III Región, 138 p.; Universidad de Chile: Santiago. Tesis de Magíster.

BOURDON, E., 2004 - A new Paleocene-Eocene avifauna from Morocco: The early diversification of the pseudo-toothed birds (Aves, Odontopterygiformes). In: Abstracts of the Sixth International Meeting of the Society of Avian Paleontology and Evolution: 9; Quillan.

BOURDON, E., 2005 -Osteological evidence for sister group relationship between pseudo-tothed birds (Aves: Odontopterygiformes) and waterfowls (Anseriformes). Naturwissenschaften, 92: 586-91; Heidelberg.

CHÁVEZ, M., 2001 - Presencia de un Pelagornithidae (Aves: Pelecaniformes) en el Mioceno de la Formación Bahía Inglesa, Tercera región, Chile. In: Resúmenes Décimo-séptimas Jornadas Argentinas de Paleontología de Vertebrados, 38 (2): 5R; Ezquel: Ameghiniana.

CHÁVEZ, M., 2005a - Nuevos registros de Aves Fósiles en la Formación Bahía Inglesa (MiocenoPlioceno), región de Atacama, Chile. In: Actas del Octavo Congreso Chileno de Ornitología: 47; Chillán.

CHÁVEZ, M., 2005b - Las Aves Fósiles de Chile y Península Antártica. In: Resúmenes del Segundo Congreso Latinoamericano de Paleontología de Vertebrados: 79-80; Río de Janeiro.

CHÁVEZ, M., 2005c - Una nueva localidad con Aves Fósiles en la región de Atacama, Chile. In: Actas del Octavo Congreso Chileno de Ornitología: 46; Chillán.

CHÁVEZ, M., 2006 - Revisión de los Pingüinos fósiles (Aves: Sphenisciformes) de la Formación Bahía Inglesa, III región, Chile. In: Resúmenes Primer Congreso Biociencias: 25; Valdivia.

CHÁVEZ, M., en prensa - Observaciones sobre la presencia de Paraptenodytes y Palaeospheniscus (Aves: Sphenisciformes) en la formación Bahía Inglesa. Revista Chilena de Historia Natural; Santiago.

CHÁVEZ, M. \& STUCCHI, M., 2002 - El registro de Pelagornithidae del Pacífico sudeste. In: Actas del Primer Congreso Latinoamericano de Paleontología de Vertebrados: 26; Santiago.

CHENEVAL, J., 1993 - L'avifaune Mio-Pliocène de la formation Pisco (Pérou) étude préliminaire. Docum. Lab. Géol., 125: 85-95; Lyon.

EMSLIE, S. \& GUERRA, C., 2003 - A new species of penguin (Spheniscidae: Spheniscus) and other birds from the late Pliocene of Chile. Proceedings of the Biological Society of Washington, 116(2): 308-316; Washington D.C.

FORDYCE, E., 1991 - A new look at the fossil vertebrate record of New Zealand. In: Vertebrate paleontology of Australasia (P. Vickers-Rich, ed.): 1191-1316; Melbourne: Pioneer Design Studio, Monash University.

FRITIS, O., 2001 - Descripción y análisis de la ornitofauna fósil de la Formación Bahía Inglesa (Mioceno superior), III región de Atacama, Chile, 43 p.; Concepción: Departamento Ciencias de la Tierra, Universidad de Concepción. Tesis de grado. 
El registro de Pelagornithidae (Aves: Pelecaniformes) y la avifauna neógena del Pacífico sudeste

GONZÁLEZ, G., GOEDERT, J. \& SCHWENNICKE, T., 2004 - First record of Osteodontornis (Aves: Pelagornithidae) from Mexico. In: Abstracts of the Sixth International Meeting of the Society of Avian Paleontology and Evolution: 26; Quillan.

GONZÁLEZ, G., SCHWENNICKE, T., GOEDERT, J. \& BARNES, L., 2002 - Earliest Pacific basin record of the Pelagornithidae (Aves: Pelecaniformes). Journal of Vertebrate Paleontology 22: 722-725; Northbrook: University of Oklahoma.

GUZMÁN, N., MARQUARDT, C., ORLIEB, L. \& FRASSINETTI, D., 2000 - La malacofauna neógena y cuaternaria del área de Caldera $\left(27^{\circ}-28^{\circ} \mathrm{S}\right)$ : especies y rangos bioestratigráficos. In: Actas del Noveno Congreso Geológico Chileno, 1: 476-481; Puerto Varas.

HARRISON, C. \& WALKER, C., 1976 - A review of the bony-toothed birds (Odontopterygiformes): with descriptions of some new species, 72 p.; Backhuys Publishers. Tertiary Research Special Paper 2.

HOWARD, H., 1980 - Illustrations of Avian Osteology taken from «The Avifauna of Emerville Shellmound». Contributions in Science, 330: xxvii-xxxviii; L.A.: Natural History Museum of Los Angeles County.

HOWARD, H. \& WARTER, S., 1969 - A new species of bony-toothed bird (Family Pseudodontornithidae) from the Tertiary of New Zealand. Records of the Canterbury Museum, 8: 345-357; New Zealand.

HOWARD, H. \& WHITE, J., 1962 - A second record of Osteodontornis, Miocene «toothed» bird. Contributions in Science, 52: 1-12; L.A.: Natural History Museum of Los Angeles County.

LAMBRECHT, K., 1930 - Studien über Fossile Riesenvögel. Geologica Hungarica, 7: 1-17; Hungary: Geological Institute of Hungary. Serie Paleontologica.

LYDEKKER, R., 1891 - Catalogue of the fossil birds in the British Museum, XII, 368 p.; London.

MARCHANT, M., MARQUARDT, C., BLANCO, N. \& GODOY, E., 2000 - Foraminíferos del área de Caldera $\left(26^{\circ} 45^{\prime}-28^{\circ} \mathrm{S}\right)$ y su utilización como indicadores cronoestratigráficos del Neógeno. In: Actas del Noveno Congreso Ceológico Chileno, 1: 499-503; Santiago.

MAROCCO, R. \& MUIZON, C. de, 1988 - Los vertebrados del Neógeno de la costa sur del Perú: ambiente sedimentario y condiciones de fosilización. Bulletin de l'Institut Français d'Études Andines, 17(2): 105-117; Lima.

McKEE, J., 1985 - A pseudodontorn (Pelecanifomes: Pelagornithidae) from the middle Pliocene of Hawera, Taranaki, New Zealand. New Zealand Journal of Zoology, 12: 181-184; New Zealand.

MOURER-CHAUVIRÉ, C. \& GERAADS, D., 2004 - The Struthionidae and Pelagornithidae (Aves: Struthioniformes and Pelecaniformes) from the late Pliocene of Ahl Al Oughlam, Morocco. In: Abstracts of the Sixth International Meeting of the Society of Avian Paleontology and Evolution: 45; Quillan.

MUIZON, C. de, 1978 - Arctocephalus (Hydractos) lomasiensis, subgen. nov. et nov. sp. un nouvel otariidae du Mio-Pliocène de Sacaco (Pérou). Bulletin de I'Institut Français d'Études Andines, 7 (3-4): 169-188; Lima.

MUIZON, C. de, 1981 - Les vertébrés fossiles de la Formation Pisco (Pérou). Première partie: deux nouveaux Monachinae (Phocidae: Mammalia) du Pliocène inférieur de Sacaco-Sud, 160 p.; París: Éditions Recherches sur les Grandes Civilisations. Mémoire 6.

MUIZON, C. de \& DEVRIES, T., 1985 - Geology and Paleontology of late Cenozoic marine deposits in Sacaco area (Peru). Geologische Rundschau, 74(3): 547-563; Stuttgart.

MUIZON, C. de, DOMMING, D. \& KETTEN, D., 2002 - Odobenocetops peruvianus, the walrus convergent delphinoid (Mammalia: Cetacea) from the early Pliocene of Peru. In: Cenozoic mammals of land and sea: Tribute to the career of Clayton E. Ray (Emry, R.-J., ed.), 92: 223-261; Smithsonian Contribution to Paleobiology.

MUIZON, C. de \& McDONALD, G., 1995 - An aquatic sloth from the Pliocene of Peru. Nature, 375: 224-227; London. 
RINCÓN, A. \& STUCCHI, M., 2005 - Primer registro de Pelagornithidae del Mioceno de Venezuela. Revista Venezolana de Espeleología, 37: 27-30; Caracas.

OLSON, S., 1985 - The fossil record of birds. In: Avian Biology (D. S. Farner, J. R. King \& K. C. Parker, eds.), 8: 79-252; Orlando: Academic Press.

OLSON, S. \& RASMUSSEN, P., 2001 - Miocene and Pliocene birds from the Lee Creek Mine, North Carolina. In: Geology and Paleontology of the Lee Creek Mine (C. Ray \& D. Bohaska, eds.), 90: 259-260; North Carolina: Smithsonian Contributions to Paleobiology.

SALLABERRY, M., RUBILAR, D., SUÁREZ, M. \& GUTSTEIN, C., 2007 - The skull of a fossil prion (Aves: Procellariiformes) fron the neogene (Late Miocene) of Northern Chile. Revista Geológica de Chile, 34: 147-154; Santiago.

SCARLETT, R., 1972 - Bone of a presumed odontopterygian bird from the Miocene of New Zealand. New Zealand Journal of Geology and Geophysic, 15: 269-274; New Zealand.

SPULSKI, B., 1910 - Odontopteryx longirostris n. Sp. Zeitschrift der Deutschen Geologischen Gesellschaft., 62: 507-521; Berlín.

STUCCHI, M., 2002 - Una nueva especie de Spheniscus de la Formación Pisco, Perú. Boletín de la Sociedad Geológica del Perú, 94: 19-26; Lima.

STUCCHI, M., 2003 - Los Piqueros (Aves: Sulidae) de la Formación Pisco, Perú. Boletín de la Sociedad Geológica del Perú, 95: 75-91; Lima.

STUCCHI, M. \& DEVRIES, T., 2003 - El registro más antiguo de Sulidae en el Perú. Boletín de la Sociedad Geológica del Perú, 96: 117-120; Lima.

STUCCHI, M. \& EMSLIE, S., 2005 - A new Condor (Ciconiiformes, Vulturidae) from the late Miocene/early Pliocene Pisco Formation, Peru. The Condor, 107 (1):107-113; Albuquerque.

STUCCHI, M. \& URBINA, M., 2004 - Ramphastosula (Aves: Sulidae): A new avian genus from the early Pliocene of the Pisco Formation, Peru. Journal of Vertebrate Paleontology, 24: 974-978; Northbrook: University of Oklahoma.

STUCCHI, M. \& URBINA, M., 2005a - Las Aves fósiles del Terciario peruano. In: Actas del Sexto Congreso Nacional de Ornitología: 134; Lambayeque.

STUCCHI, M. \& URBINA, M., 2005b - Historia evolutiva de los pingüinos (Spheniscidae) fósiles de la costa peruana: Primeros alcances. In: Actas del Sexto Congreso Nacional de Ornitología: 135; Lambayeque.

STUCCHI, M. \& URBINA, M., 2005c - Nuevos restos de Procellariiformes fósiles de la formación Pisco, Perú. Boletín de la Sociedad Geológica del Perú, 100: 67-77; Lima.

STUCCHI, M. \& URBINA, M., 2005d - Evidence of a Fossil Stork (Ciconiidae) from the Late Miocene of the Pisco formation, Peru. Boletín de la Sociedad Geológica del Perú, 100: 63-66; Lima.

STUCCHI, M., URBINA, M. \& GIRALDO, A., 2003 - Una nueva especie de Spheniscidae del Mioceno tardío de la Formación Pisco. Boletín del Instituto Francés de Estudios Andinos 32 (2): 361-375; Lima.

SUÁREZ, M., CHÁVEZ, M. \& MARQUARDT, C., 2002 - Nuevos hallazgos de vertebrados marinos de la Formación Bahía Inglesa (Mioceno-Plioceno) Caldera, Norte de Chile. In: Actas del Primer Congreso Latinoamericano de Paleontología de Vertebrados: 50-51; Santiago.

SUÁREZ, M. \& MARQUARDT, C., 2003 - Revisión preliminar de las faunas de peces elasmobranquios del Mesozoico y Cenozoico de Chile y comentarios sobre su valor cronoestratigráfico; Concepción. Tercera sesión temática del Décimo Congreso Geológico Chileno. CD-rom.

TAMBUSSI, C., ACOSTA, C. \& CANTO, J., 2005 - Paleornitofauna de pingüinos de Chile. In: Resúmenes del Segundo Congreso Latinoamericano de Paleontología de Vertebrados: 259-260; Río de Janeiro. 
El registro de Pelagornithidae (Aves: Pelecaniformes) y la avifauna neógena del Pacífico sudeste

TONNI, E., 1980 - Un Pseudodontornitido (Pelecaniformes, Odontopterygia) de gran tamaño, del Terciario temprano de Antártida. Ameghiniana, 17(3): 273-276; Buenos Aires: Asociación Paleontológica Argentina.

TONNI, E. \& CIONE, A., 1978 - Una nueva colección de vertebrados del Terciario inferior de la isla Vicecomodoro Marambio (Seymour Island), Antártida: 73-79; Argentina. Obras Centenario Museo de la Plata 5.

TONNI, E. \& TAMBUSSI, C., 1985 - Nuevos restos de Odontopterygia (Aves: Pelecaniformes) del Terciario temprano de Antártida. Ameghiniana, 21(2-4): 121-124; Buenos Aires: Asociación Paleontológica Argentina.

URBINA, M. \& STUCCHI, M., 2005 - Los Cormoranes (Aves: Phalacrocoracidae) de la Formación Pisco, Perú. Boletín de la Sociedad Geológica del Perú, 99: 41-49; Lima.

WALSH, S., 2000 - Big-chested birds-exciting new avian material from the Neogene of Chile. In: 48th Symposium of Vertebrate Palaeontology and Comparative Anatomy; Portsmouth. http://www.svpca.org/years/2000_portsmouth/abstracts.php

WALSH, S., 2004 - New penguin remains from the Neogene of Chile. In: Abstracts of the Sixth International Meeting of the Society of Avian Paleontology and Evolution: 60-61; Quillan.

WALSH, S. \& HUME, J., 2001 - A new neogene marine avian assemblage from north-central Chile. Journal of Vertebrate Paleontology, 21: 484-491; Northbrook: University of Oklahoma.

WALSH, S. \& SUÁREZ, M., 2006 - New penguin remains from the Pliocene of northern Chile. Historical Biology, 18: 115-126.

WARHEIT, K., 2002 - The seabird fossil record and the role of paleontology in undestanding seabird community structure. In: Biology of marine birds (E. Schreiber \& J. Burger, eds.): 17-55; Florida: CRC Press. 